\section{El estado de las lesiones causadas por el tránsito en México: evidencias para fortalecer la estrategia mexicana de seguridad vial}

\author{
Road traffic injuries in Mexico: evidences to \\ strengthen the Mexican road safety strategy \\ Lesões por acidentes de trânsito no México: \\ evidências para fortalecer a estratégia de \\ segurança rodoviária mexicana
}

Ricardo Pérez-Núñez 1

Martha Hijar 1,2

Alfredo Celis 3

Elisa Hidalgo-Solórzano ${ }^{1}$

\author{
${ }^{1}$ Centro de Investigación en \\ Sistemas de Salud, Instituto \\ Nacional de Salud Pública, \\ Cuernavaca, México. \\ 2 Fundación Entornos A.C. \\ Cuernavaca, México. \\ 3 Centro Universitario \\ de Ciencias de la \\ Salud, Universidad de \\ Guadalajara, Guadalajara, \\ México. \\ Correspondencia \\ R. Pérez-Núñez \\ Centro de Investigación en \\ Sistemas de Salud, Instituto \\ Nacional de Salud Pública. \\ Universidad No. 655, Col. \\ Santa María Ahuacatitlán. \\ Cerrada, Cuernavaca I \\ Morelos-62100, México. \\ ricardo.perez@insp.mx
}

\begin{abstract}
Based on a review of secondary data and the scientific literature and an analysis of the ENSANut-2012 database, the current study provides a comprehensive overview of the current burden of road traffic injuries (RTI) in Mexico and analyzes the country's social response to RTI. The high collision, injury, mortality, and disability rates associated with this public health problem represent a high cost for Mexican society, especially for families. The paper argues that the Mexican response has focused on vehicle occupants while overlooking vulnerable road users and has prioritized strategies with limited effectiveness. Although the country's existing legislation addresses the main risk factors, enforcement has been limited. Finally, the paper makes some recommendations for strengthening the Mexican strategy to prevent RTI, such as safe, equitable, healthy, and sustainable mobility for all types of road users. Despite some strides in RTI prevention, there are still challenges and opportunities to be addressed in the future.
\end{abstract}

Traffic Accidents; Health Public Policy; Health Surveys

\section{Resumen}

Mediante la revisión de información secundaria oficial, publicaciones científicas y el análisis de la ENSANut-2012, este trabajo busca proveer una visión integral de la magnitud actual de las lesiones causadas por el tránsito (LCT) en México y analizar críticamente la respuesta social organizada. Las altas tasas de choques, lesiones, muertes y discapacidad atribuidas a este problema de salud pública, representan un elevado costo para la sociedad mexicana, en especial para los hogares. Se discute cómo la respuesta mexicana se ha enfocado a los automovilistas, dejando fuera a usuarios vulnerables de la vía pública y se ha privilegiado la implementación de estrategias poco efectivas. A pesar de que se dispone de legislación para los principales factores de riesgo, se ha descuidado su correcta aplicación. La estrategia mexicana podría fortalecerse implementando estrategias de movilidad seguras, equitativas, saludables y sostenibles para los diferentes actores de la vía pública. A pesar de los avances que se han dado en términos de prevención de LCT, existen aún retos y oportunidades para el país.

Accidentes de Tránsito; Políticas Públicas de Salud; Encuestas Epidemiológicas 


\section{Introducción}

El reciente estudio de la carga de la enfermedad muestra cómo de 1990 a 2010 las lesiones causadas por el tránsito (LCT) en el mundo se incrementaron un 46,3\% para llegar a 1,3 millones de muertes por año. Esto implicó un incremento en la tasa de mortalidad de $6,2 \%$, pasando de 18,4 a 19,5 por 100 mil habitantes. Este incremento fue mayor en peatones $(62,3 \%$ en las muertes y $17,6 \%$ en la tasa), motociclistas $(56,7 \%$ en muertes y $14,4 \%$ en la tasa) y ciclistas $(51,7 \%$ muertes, $7,8 \%$ en la tasa de mortalidad). Así de ser la décima causa de muerte en 1990 pasó a ser la octava en 2010 1. Estimaciones de la Organización Mundial de la Salud (OMS) proyectan que de seguir la tendencia actual, se constituirá como la quinta causa de muerte en 20302.

Las LCT son un fenómeno complejo y de causas múltiples ${ }^{3}$. La OMS ha puesto gran énfasis en cinco factores de riesgo sobre los cuales considera es imperativo trabajar, en aras de lograr la seguridad vial: consumo de alcohol y conducción, no uso de cinturón de seguridad y sistemas de retención infantil (SRI), no uso de casco en motociclistas y velocidad inadecuada 4 . Hay, sin embargo, otros factores de riesgo que han recibido poca atención.

Pocas evidencias han sido publicadas en México que analicen desde una perspectiva integral el problema de las LCT. Conscientes de que un enfoque, basado en evidencias, para la prevención y atención de las lesiones puede y debe adoptarse 5, el objetivo del presente estudio fue utilizar las evidencias disponibles para hacer un diagnóstico de la situación actual de las LCT en el país y analizar la respuesta social a este problema. A partir de este diagnóstico, se establecen recomendaciones de política pública orientadas a reforzar la estrategia mexicana de seguridad vial. Consideramos que el análisis del caso mexicano podría servir de insumo para la reflexión y discusión de investigadores y tomadores de decisiones en otros contextos.

\section{Metodología}

Mediante una revisión narrativa, se analiza tanto información primaria como secundaria. La información primaria proviene de la Encuesta $\mathrm{Na}$ cional de Salud y Nutrición 2012 (ENSANut-2012) que es representativa para el ámbito nacional y estatal y constituye la información a escala nacional más reciente en el tema. Detalles sobre su diseño han sido publicados previamente ${ }^{6,7}$. Algunos resultados aquí presentados se publicaron en una ficha técnica como estrategia de difusión rápida 8 . De igual manera, se analizó información secundaria proveniente de tres tipos de fuentes:

- Sistemas de información oficiales disponibles para el ámbito nacional sobre: mortalidad, egresos hospitalarios, adicciones, urgencias y servicio médico-forense;

- Documentos oficiales de las dependencias públicas encargadas de dar respuesta al problema de las LCT;

- Artículos publicados en MEDLINE/PubMed, publicados de 1990 a enero de 2013, que reportan información sobre la epidemiología de las LCT en México, sus determinantes y consecuencias asociadas; intervenciones, programas, políticas y respuesta social organizada a este problema de salud pública. Esta revisión fue realizada por dos investigadores de forma independiente y luego se reunieron para integrar ambos abordajes. Se identificaron inicialmente 93 publicaciones, 37 (40\%) fueron eliminadas por no coincidir con los objetivos de este estudio (temas distintos a las LCT, realizados en otro país, trabajos clínicos o biomédicos). De los 56 seleccionados, se eliminaron 9 artículos de revisión, comentarios, reporte de casos y editoriales -a partir de la revisión del resumen- y 15 tras leer el trabajo completo y observar que no aportaban información adicional. Cuando formaban parte del mismo estudio o abordaban el mismo tema se incluyó sólo el más reciente. Finalmente, se incluyeron 32 publicaciones de relevancia para este estudio.

El análisis se contextualizó a la luz de la evidencia internacional.

\section{Resultados}

Primero se presenta el análisis sobre la epidemiología de las LCT en México, a continuación se aborda la exposición a los principales factores de riesgo documentados; posteriormente, se analizan las consecuencias económicas y sociales documentadas; finalmente se presenta la respuesta social al problema de las LCT.

\section{Epidemiología de las LCT en México}

De 1999 a 2010 fallecieron casi 185 mil personas por LCT. De 1999 a 2009 la tasa de mortalidad presentó un incremento de $1,5 \%$ por año ${ }^{9}$ hasta llegar a una tasa de 16,6 por 100 mil habitantes lo que implica que, en ese año, 17.851 personas perdieron la vida por esta causa. Para 2010 el número de muertos se redujo $7 \%$ para un total de 16.595 y una tasa de 15,3 por 100 mil habitantes (Dirección de Información en Salud, Secretaría de Salud. Sistema Nacional de Información en Salud. http://www.sinais.salud.gob.mx/estadis 
ticasportema.html, accedido el 17/May/2012). Sin embargo, estas altas cifras podrían estar subestimadas en alrededor de un $30 \%$, si se considera el número de personas cuya causa de muerte no es bien registrada o es clasificada en códigos inespecíficos 9,10,11.

Las LCT constituyen la séptima posición entre las causas de mortalidad específica en México. De igual forma, representan la primera causa de muerte en el grupo de 5-15 años y la cuarta causa de muerte en población de 15-64 años de edad. En los hombres, las muertes en eventos de tránsito ocupan el quinto lugar (Dirección de Información en Salud, Secretaría de Salud. Sistema Nacional de Información en Salud. http:// www.sinais.salud.gob.mx/estadisticasportema. html, accedido el 17/May/2012). Cerca del 65\% de estas muertes ocurren entre los 20 y 59 años de edad. Sin embargo, el grupo de edad con la mayor tasa de mortalidad es el de las personas de 60 y más años ( 40 por cada 100 mil personas de 60 y más) 9,11. Las LCT son el tipo de lesiones no intencionales que generan el mayor número de víctimas mortales 12 y son las de mayor gravedad 13,14 .

Entre los usuarios de la vía pública que más fallecen por esta causa se encuentran los peatones que representan entre $45-48 \%$ del total, y los ocupantes de vehículo automotor con un $37-48 \%$. Es de resaltar que la tasa de mortalidad de motociclistas se incrementó un 332,2\% entre 1999 y 2009 9,11. En este mismo periodo el número de motocicletas en México aumentó un $312 \%$ para llegar a una tasa de 1.114 motocicletas por 100 mil habitantes (Instituto Nacional de Estadística y Geografía. Estadística de Vehículos de Motor Registrados en Circulación. Anual, 1999-2009. http://www.inegi.org. $\mathrm{mx} /$ sistemas/olap/proyectos/bd/consulta. asp?p=14745\&c=23717\&s=est\&cl=4, accedido el 15/Ene/2013). Es posible que en el futuro los motociclistas se conviertan en un grupo de mayor peso relativo en términos de morbi-mortalidad. De ahí que la OMS ha llamado la atención sobre las necesidades particulares de los usuarios de la vía pública más vulnerables: peatones, ciclistas, motociclistas; pues representan en el ámbito mundial $50 \%$ de todas las personas que fallecen por el tránsito en el mundo. En México, cerca de $60 \%$ de los fallecidos podrían pertenecer a estos usuarios vulnerables 9 .

El problema de las LCT no sólo lo constituye el alto número de personas que fallecen por esta causa. Para 2006, prácticamente 1,03 millones de personas reportaron haber sufrido LCT 15. Esto implica, que alrededor de 65 personas se lesionaron durante 2006 por cada persona que fallece. Datos de la ENSANut-2012, muestran que el nú- mero de personas que reportaron haber sufrido LCT ascendió a 1,4 millones para 2012 7. El incremento fue más evidente en hombres quienes tuvieron una prevalencia de un 1,6\%, mayor a la reportada en 2006 y 2000 de un 1,3\% y un 1,2\%, respectivamente. Los hombres entre 20-34 años presentaron la mayor prevalencia.

Hasta antes de la ENSANut-2012 poco se sabía sobre la prevalencia de LCT no-fatales, según tipo de usuario. Del total de LCT, un $47 \%$ fueron usuarios vulnerables: un $23 \%$ motociclistas, un $13 \%$ peatones y $12 \%$ ciclistas. Es importante mencionar que los atropellamientos fueron el tipo de LCT más prevalente en adultos mayores (37\%) y en menores de 9 años (38\%).

A partir de la ENSANut-2012 se estimó que 320.496 personas fueron hospitalizadas por LCT durante el año previo a la encuesta, 7,2\% del total de hospitalizaciones -cifra mayor a los 71-77 mil egresos estimados previamente 16. En promedio, cada lesionado dura entre 5 y 6 días hospitalizado 17 , pierde 26 días 18 y $43 \%$ de los lesionados tarda en promedio un año para reincorporarse a su actividad laboral 15. Además, 382.113 personas utilizaron los servicios ambulatorios durante los 15 días previos a la encuesta, presumiblemente por LCT, constituyendo el $4,1 \%$ del total de usuarios 7 .

\section{Exposición a los principales factores de riesgo}

A continuación se presenta la evidencia documentada para el contexto nacional sobre algunos de los principales factores de riesgo (la Figura 1 hace un recuento de la evidencia internacional).

\section{- Alcohol}

En cuanto a la prevalencia de uso de alcohol y conducción, alrededor de un $20 \%$ de los conductores a quienes se les realizó una alcoholimetría dieron positivo, $3 \%$ estuvieron por arriba de los límites permitidos 19. También el alcohol está presente en los eventos de tránsito: $10 \%$ de los conductores responsables de choques registrados en vías urbanas y suburbanas de 1999 a 2010 fue reportado con aliento alcohólico (Instituto Nacional de Estadística y Geografía. Accidentes de Tránsito Terrestre en Zonas Urbanas y Suburbanas (ATUS). Anual, 1999-2012. http://www.inegi. org.mx/sistemas/olap/proyectos/bd/consulta. asp? $\mathrm{p}=14744 \& \mathrm{c}=23705 \& \mathrm{~s}=\mathrm{est} \& \mathrm{cl}=4$, accedido el 15/Nov./2013). Esta proporción disminuyó de $13,7 \%$ en 1999 a 7,8\% durante 2010. Alrededor de un 1,6\% de los eventos de tránsito reportados en carreteras federales fueron causados por estado de ebriedad del conductor 20 . 
Figura 1

¿Qué sabemos de...

\section{...la conducción bajo la influencia del alcohol?}

- Las concentraciones de alcohol en sangre por encima de $0,04 \mathrm{~g} / \mathrm{dL}$ incrementan el riesgo de que un conductor se vea involucrado en un siniestro vial 4

...del uso de dispositivos de retención?

- Existen evidencias que muestran que los cinturones de seguridad reducen en un 40-50\% el riesgo de fallecer tras una colisión para los pasajeros de adelante y un $25-75 \%$ para los de atrás 75,76 ;

- Los sistemas de retención infantil, cuando son instalados, y utilizados de forma correcta, pueden reducir las muertes de tránsito en un $70 \%$ en menores de un año y en 54-80\% en menores de 1-4 años 77 .

...del uso de casco?

- Los cascos reducen en un $69 \%$ las lesiones de cabeza en motociclistas que colisionan y la muerte en un $42 \% 78$ y sufren menos lesión cervical que aquéllos que no lo utilizan 79;

- El uso de casco reduce el riesgo de traumatismos craneoencefálicos en ciclistas entre un $63 \%$ y $88 \%$ 4;

- Utilizar un casco no certificado está asociado a una mayor prevalencia y severidad de lesiones de cráneo cuando se compara con personas que usan casco certificado e incluso con aquéllas que no utilizan casco 80 .

...de la velocidad excesiva o inadecuada?

- A mayor velocidad aumenta la probabilidad de ocurrencia de colisiones, así como la severidad de las lesiones causadas por este tipo de eventos 4;

- La reducción de los límites de velocidad reduce la frecuencia y severidad de las lesiones de tránsito en usuarios vulnerables como peatones, ciclistas y motociclistas 32,64 .

...del uso de celular?

- Diversos estudios han revelado que el uso de telefonía móvil al conducir incrementa el riesgo de choques hasta en cuatro veces 81 y que el riesgo no disminuye con el uso de "dispositivos de manos libres" 81,82;

- En Estados Unidos se ha documentado que las muertes causadas por este y otro tipo de distracciones al conducir ha incrementado un $28 \%$ de 2005 a 2008 , pasando de 4.572 a 5.870 83;

- También se ha documentado cómo el uso de celular al deambular afecta la seguridad de los peatones 84 .

...del diseño de la estructura vial y del medio ambiente?

- La creación y modificaciones de la estructura vial tienen consecuencias directas sobre la salud de la población 32

- Una estrategia efectiva para lograr una movilidad segura es la correcta separación de espacios entre los diferentes usuarios de la vía pública ${ }^{85}$;

- Algunos factores ambientales identificados como de riesgo para el atropellamiento en niños son: el volumen vehicular, el número de vehículos estacionados en las calles, límites de velocidad mayores, así como cuando hay mayor número de peatones o densidad poblacional de niños. Sitios con mayor flujo vehicular, densidad habitacional y casas multifamiliares, o con la presencia de hacinamiento y ausencia de áreas de juegos para niños, así como sitios con menores ingresos y altas tasas de desempleo, están asociados con mayores prevalencias de atropellamientos $57,58,86$. Por el contrario, cuando el porcentaje de hogares con ingresos superiores es mayor, la prevalencia de atropellamientos es menor 86 .

El Sistema de Vigilancia Epidemiológica de las Adicciones reporta que un 9\% de los lesionados que utilizaron servicios de urgencias y un $20 \%$ de las personas que fallecieron en México por esta causa, de 1994 a 2010, habían consumido alcohol (Dirección General de Epidemiología. Sistema de Vigilancia Epidemiológica de las Adicciones (SISVEA), 1994-2011. http:///www.epide miologia.salud.org.gob.mx/dgae/infoepid/inf sisvea.html, accedido el 11/Feb./2014). Un estudio realizado en Nuevo León encontró que un $49 \%$ de los conductores y un $32 \%$ del total de fallecidos en el tránsito tenían alcohol en sangre 21. Resultados similares fueron obtenidos en otro estudio realizado en la carretera México-Cuer- navaca 22, en donde además se documentó que el alcohol también está asociado a una mayor severidad ${ }^{23}$. También en peatones se ha observado que un alto porcentaje refiere haber ingerido bebidas alcohólicas antes de ser atropellado 24 .

En la ENSANut-2006, de 264.823 adolescentes (10-19 años de edad) que presentaron LCT, entre $1-7 \%$ reportaron estar bajo el influjo del alcohol en el momento del siniestro vial 25 . En la ENSANut-2012, un 5,9\% de los adolescentes (IC95\%: 3,2-10,5\%) y un 9,2\% (IC95\%: 5,3-15,7\%) de los adultos con LCT reportaron estar bajo el influjo del alcohol. Al analizar esto por tipo de usuario vial, el uso de alcohol fue más frecuente en ocupantes de vehículos automotores $(10,16 \%$; 
IC95\%: 5,29-18,62\%), seguido de ciclistas (7,62\%; IC95\%: 2,26-22,78\%), motociclistas (6,46\%; IC95\%: $3,02-13,28 \%)$ y peatones $(2,67 \%$; IC95\%: $0,58-11,51 \%)$.

\section{- No uso de cinturón y SRI}

A pesar de ser una estrategia costo efectiva, en México existe poca información que documente el uso de estos dispositivos de retención. Sólo un $25 \%$ de los conductores que colisionaron durante 2010 en zonas urbanas y suburbanas utilizaban el cinturón de seguridad en el momento del siniestro. El uso de cinturón de seguridad se ha estimado en un $45 \%$ en varios municipios del país 19,26 y el porcentaje es aún menor (31\%) entre lesionados usuarios de los servicios de salud 26 . El uso de SRI se ha estimado en alrededor de un $6,8 \%$ en menores de 10 años y de entre 7,9 a $17,4 \%$ en menores de 5 años 26 y se ha visto que su uso tiende a ser menor en estratos socioeconómicos bajos 27 .

La ENSANut-2012 muestra que un $44,9 \%$ (IC95\%: 36,6-53,5\%) de las personas de 10 y más años reportó utilizar cinturón de seguridad en el momento de la colisión. Por otro lado, un 12,3\% (IC95\%: 3,6-34,7\%) de los lesionados menores de 10 años utilizaban SRI.

\section{- No uso de casco}

El uso de casco reportado en el país oscila entre 68 y $99 \% 19$ y se ha observado que los conductores lo utilizan más que los pasajeros 28 . Sin embargo, un estudio realizado en Cuernavaca muestra que un $65 \%$ de los cascos utilizados no están certificados 29. A partir de la ENSANut-2012 podemos observar que el uso de casco en motociclistas lesionados en México fue de un 55,3\% (IC95\%: $43,3-66,7 \%)$. El uso de casco por ciclistas es menor: un 9,8\% (IC95\%: 3,4-25,2\%) de lesionados reportaron su uso en el momento del evento.

\section{- Velocidad excesiva o inadecuada}

Pocas evidencias existen en México, en relación a la exposición a este importante factor de riesgo. Un estudio realizado en una autopista del país documentó que entre un 14,7 y 20,1\% de los vehículos que colisionaron sobrepasaba el límite de velocidad 22. Reportes oficiales atribuyen un $44,3 \%$ de las colisiones en carreteras federales a la velocidad excesiva 20 ; sin embargo, esta cifra podría no estar sustentada en evidencias.

\section{- Uso de celular}

Estudios recientes estiman que, durante 20112012, un $10,78 \%$ y un $0,64 \%$ de un total de 7.940 automovilistas 30 y 4.244 motociclistas 31 , respectivamente, que fueron observados aleatoriamente en tres ciudades del país, utilizaban el teléfono móvil al conducir.

\section{- Diseño y adecuación de la estructura vial e invasión de espacios}

Las políticas públicas, derivadas del crecimiento de las grandes ciudades en nuestro país, han promovido el traslado de personas en vehículos privados 32 . Así, se ha observado cómo el diseño de la estructura vial se ha convertido en un espacio de mayor peligro para los peatones, que son los usuarios más numerosos y vulnerables 24 . De entre los peatones, la inadecuación de la estructura vial pone en mayor riesgo a los menores de 1-14 años 33, a los adultos mayores y a las personas con algún tipo de discapacidad. La estructura vial es igualmente insegura para el creciente número de ciclistas y motociclistas que circulan en el país.

En nuestro país se ha documentado la invasión de espacios peatonales por vehículos de motor. Los automovilistas, y el transporte público, se detienen en lugares no permitidos y no respetan las señales de tránsito. Los vendedores ambulantes se apropian de las banquetas para ejercer su actividad, desplazando y poniendo en más riesgo a los peatones que se ven forzados a deambular por las calles 34 . Los vendedores ambulantes están también en riesgo por su autoexposición a la invasión de espacios, que cuando fueron diseñados no se consideró la presencia de esa actividad comercial 24 .

También los peatones invaden los espacios destinados para otros usuarios cuando cruzan, de forma insegura, una vía o cuando abordan o descienden del transporte público en sitios inadecuados 24 . Poco más de un $50 \%$ de los peatones no utilizan los puentes peatonales 35. Entre las razones de no uso está la imposibilidad de algunos actores de utilizarlos (adultos mayores y personas con discapacidad). Además, los adultos mayores, presentan limitaciones visuales y motoras que les impide desplazarse con seguridad 24 . Las personas con discapacidad tienen un riesgo mayor de sufrir LCT como peatones, ciclistas o usuarios de sillas de ruedas, lo que se ha llamado el doble riesgo de las personas que viven con discapacidad 36 .

Un sistema que privilegia el uso de la vía pública para el transporte privado, no favorece que los diferentes usuarios reconozcan este espacio 
común. Esto, aunado a un bajo nivel de compromiso cívico y escasez de recursos sociales, disminuye la posibilidad de lograr la participación social en el control de los espacios y de las LCT 37.

\section{Consecuencias económicas y sociales} de las LCT

Las LCT imponen una fuerte carga económica para la sociedad 25. Un alto número de personas utilizan servicios de salud ambulatorios o son hospitalizados por esta causa. Esto, junto con la pérdida de productividad, asociada al tiempo que tardan los lesionados para reincorporarse a su actividad laboral, genera altos costos directos e indirectos. Así, el costo económico de las LCT para el Sistema de Salud fue de US\$ 7,598 millones durante 2007, lo que representa el $1 \%$ del PIB nacional de ese mismo año ${ }^{38}$. Paralelamente, el costo económico total de los eventos de tránsito ocurridos sólo en carreteras federales durante 2009 se estimó oficialmente en US\$2,440 millones, lo que incluye el costo asociado a daños a la propiedad 20 .

En el interior de los hogares las repercusiones son graves: un $8 \%$ de los lesionados atendidos en urgencias, y $80 \%$ de los hospitalizados, incurre en gastos catastróficos por su atención médica 39 . Esto repercute directamente en la economía familiar, pues con frecuencia se deja de percibir el más importante -y comúnmente el único- ingreso del hogar, traduciéndose en problemas de abandono escolar y perpetuación de los círculos de pobreza 40 . Las consecuencias económicas de estos eventos sumergen a algunos hogares a un estrés constante, pues las deudas comúnmente se prolongan al mediano y largo plazo. En algunos casos, generan dependencia económica de los hogares, afectando su sostenibilidad 41 . De ahí que no todo el costo asociado a este problema puede traducirse fácilmente a un valor monetario. Se sabe que las muertes causadas por el tránsito constituyen la segunda causa de orfandad en México 42 y que estas experiencias suelen cambiar el estado de salud de las personas, afectando las relaciones intrafamiliares y en casos extremosos destruyendo núcleos familiares previamente consolidados 41 .

Según la ENSANut-2012 de los 10 millones de personas que viven con discapacidad en México, $12,3 \%$ (11,1-13,6\%) fue causada por una lesión de tránsito. Asimismo, un 16,4\% (2,8-20,9\%) de los lesionados en el tránsito reportó consecuencias permanentes en su estado de salud, lo que se traduce en un total de 226.591 nuevas personas al año con algún grado de discapacidad. El porcentaje de lesionados menores de 10 años que fueron reportados con consecuencias permanentes se incrementó de un 3,5\% (1,4-8,2\%) en la Encuesta Nacional de Salud de 2000 (ENSA-2000) 18 a un $16,8 \%(12,8-20,9 \%)$ en la ENSANut-2012.

Esto proporciona argumentos sólidos para que los tomadores de decisiones gestionen la asignación de mayores recursos destinados a la implementación de estrategias de prevención o limitación del daño. Actualmente, el alto costo económico de este problema contrasta con la baja inversión que el Estado Mexicano emplea para su prevención. En 2008, el gobierno asignó 96 millones de pesos para financiar la estrategia mexicana para la seguridad vial ${ }^{32}$. Esto representa el $0,12 \%$ del costo económico de las LCT para 2007. Además de asignar mayores recursos financieros, se debe privilegiar la implementación de estrategias costo-efectivas y la generación de información útil para retroalimentar la toma de decisiones.

\section{La respuesta social a las LCT en México}

Diversas estrategias han sido implementadas en el país para responder a este problema de salud pública (Figuras 2 y 3). A continuación planteamos algunas de ellas.

\section{- Informar, capacitar, educar}

Dado que la mayor parte de los abordajes para el estudio de las LCT se ha centrado en los individuos y sus características, se han logrado identificar distintos factores que los ponen en mayor riesgo de chocar, lesionarse y morir. Gran parte de ellos ha concluido que es el error humano la causa de la mayoría de ellos 43 . De ahí que un número importante de intervenciones y programas de salud se focalicen en grupos considerados de mayor riesgo 44,45. La mayor parte buscan el cambio de conductas de riesgo, brindando información a la población sobre los efectos negativos de dichas conductas o sobre los beneficios de utilizar los dispositivos de seguridad existentes. Este tipo de intervenciones asume que las personas con información se conducirán racionalmente.

El problema es que aquellos en riesgo no perciben en su conducta un problema, por lo que resulta ingenuo intentar cambiar su forma de actuar, pues la gente actúa por recompensas personales inmediatas 46. Gran parte de los estilos de vida son dictados por normas sociales, de ahí que es difícil lograr que la gente se comporte diferente de sus pares ${ }^{47}$. La conducta humana no está solamente gobernada por el conocimiento y las habilidades individuales, sino por el ambiente en el cual la conducta toma lugar 48,49 . La utilización de la información y la publicidad por sí solas son generalmente insuficientes en la reducción 
Figura 2

La Iniciativa Mexicana de Seguridad Vial (IMESEVI).

La IMESEVI representa un esfuerzo de la Secretaría de Salud Federal que busca reducir las lesiones, discapacidad y muertes causadas por el tránsito en el país. Este programa, que recibió financiamiento de la Filantropía de Bloomberg, se inició durante 2008 en 4 municipios y actualmente se enfoca en 6 ciudades prioritarias y se ha extendido a diferentes ciudades y estados de la república mexicana. La IMESEVI se cimentó en un diagnóstico que evidenciaba los esfuerzos aislados de diferentes instituciones y sectores para un problema multicausal y multifactorial que supone coordinar esfuerzos para el objetivo conjunto de prevenir las lesiones causadas por el tránsito.

La IMESEVI promueve acciones para disminuir la conducción bajo influencia del alcohol e incrementar el uso de dispositivos de seguridad, entre otras. Para ello, se llevan a cabo estrategias de comunicación social, foros y talleres sobre seguridad vial para la población en general -especialmente para grupos prioritarios como los jóvenes-, promoción de modificaciones a los marcos legales vigentes, capacitación de responsables de la seguridad vial, auditorías de seguridad vial y elaboración y distribución de material gráfico informativo y de promoción de seguridad vial 19

Los resultados positivos que se documentaron para esta primera etapa motivaron a la Fundación Bloomberg a continuar con una segunda etapa en la que se incluyeron otros países de bajos y medianos ingresos para el periodo 2010-2014. Así es como nace el proyecto RS-10 (Road Safety in 10 Countries). El RS-10 en México incluyó inicialmente dos ciudades (Guadalajara y León) y consiste en la realización de puestos de control para la realización de alcoholimetrías, campañas de comunicación social y actividades educativas en jóvenes. En Guadalajara la intervención incluye la reducción en la legislación de los niveles tolerados de alcohol al conducir a 0,05g/dL, así como un incremento en las multas correspondientes. Pese a que en ambas ciudades los componentes específicos pueden variar, en las dos se enfocan a los factores de riesgo de conducir bajo los efectos del alcohol y el no-uso de cinturón de seguridad y dispositivos de retención infantil. Estas intervenciones se centran principalmente en automovilistas, aunque la estrategia de alcohol y conducción pudiera tener externalidades positivas para la seguridad de otros usuarios que comparten la vía.

Figura 3

El Decenio de Acción por la seguridad vial y la Estrategia Mexicana de Seguridad Vial.

La ONU, a través de la OMS, ha hecho un llamamiento a los países para reforzar globalmente las acciones por la seguridad vial. Así, durante 2011 se proclamó el periodo 2011-2020 como el Decenio de Acción para la seguridad vial. La estrategia del decenio de acción se sustenta sobre cinco pilares: gestión de la seguridad vial, vías de tránsito y movilidad más seguras, vehículos más seguros, usuarios de vías de tránsito más seguros, respuesta adecuada y oportuna cuando estos eventos se presenten.

México, al igual que gran parte de los países del mundo, se sumó a esta iniciativa. En la Estrategia Nacional por la Seguridad Vial 87, el país se comprometió a reducir 50\% las muertes, así como reducir al máximo posible las lesiones y discapacidades secundarias a eventos de tránsito. La Estrategia Nacional de Seguridad Vial establece la participación de los tres niveles de gobierno y su rendición de cuentas, lo que constituye un paso importante para el país. Este acuerdo fue firmado por la Secretaría de Comunicaciones y Transporte y la Secretaría de Salud. La Estrategia Nacional de Seguridad Vial plantea implementar una serie de acciones, entre las que se encuentran:

1. Coadyuvar al fortalecimiento de la capacidad de gestión de la seguridad vial;

2. Participar en la revisión de la modernización de la infraestructura vial y de transporte más segura;

3. Fomentar el uso de vehículos más seguros;

4. Mejorar el comportamiento de los usuarios de las vialidades, incidiendo en los factores de riesgo que propician la ocurrencia de eventos de tránsito;

5. Fortalecer la atención del trauma y de los padecimientos agudos, mediante la mejora de los servicios de atención pre-hospitalaria y hospitalaria.

OMS: Organización Mundial de la Salud; ONU: Organización de las Naciones Unidas. 
de eventos de tránsito ${ }^{3}$, observando, incluso, que este tipo de intervenciones suelen generar inequidad en términos de los beneficios potenciales que ofrecen 50 . Las campañas televisivas que promueven conductas saludables en automovilistas cada periodo vacacional siguen siendo parte fundamental de la estrategia nacional, sin que quede claro cuál es el impacto qué tienen en prevenir LCT o cambiar conductas de riesgo 3 .

Tampoco existe evidencia concluyente de que los programas educativos focalizados sean efectivos 51 . Esta estrategia podría ser el primer paso para el establecimiento de un vínculo con los incentivos y la coerción de los actores viales 15. Una de las cosas que la salud pública ha aprendido es que la educación para la salud, a nivel individual, es una pérdida de tiempo cuando es de una sola ocasión. Para tener resultados se requiere una inversión considerable de tiempo de consejería con refuerzos constantes, lo que además de ser costoso 47 , suele tener poco alcance en la población general. De ahí que los cambios, cuando se presentan, suelen verse en los recambios generacionales 52 .

\section{- Fabricación de autos más seguros}

En un escenario ideal, el "error humano" no tendría que traducirse en lesiones y muerte. Los cinturones de seguridad, los SRI, las bolsas de aire, el dispositivo de absorción de energía en colisiones de vehículos de motor, los muros de contención ergonómicos, las rampas de frenado, los amortiguadores de impacto, los pretiles, han sido creados con el fin de ofrecer protección pasiva a los distintos usuarios de la vía pública. Otras tecnologías de seguridad activa como sistemas de frenado antibloqueo, sistemas de control de la estabilidad, sistema de dirección y suspensión de los vehículos automotores, la iluminación, la tecnología de los neumáticos e incluso los recordatorios para el uso de cinturón de seguridad contribuyen a evitar o reducir la magnitud de los choques.

La introducción de estos dispositivos de seguridad en México ha sido principalmente por iniciativa de la industria automotriz. De ahí que la disponibilidad de estos dispositivos incrementa los precios de mercado, lo que hace que el intervalo de tiempo entre que los vehículos disponen de esta tecnología y cuando terminan reemplazando a aquéllos que no la tienen sea bastante amplio.

Salvo en el caso de cinturones de seguridad, México poco había hecho para establecer requisitos mínimos de seguridad en automóviles. Es hasta la estrategia mexicana de seguridad vial que este tema es abordado. Por otro lado, el dise- ño de las motocicletas ha evolucionado sin tener en cuenta la seguridad de sus ocupantes, por lo que es imperativo regular estándares de seguridad óptimos que redunden en una mayor seguridad de estos usuarios vulnerables 53 .

\section{- Construcción de vialidades más seguras}

La identificación de factores de riesgo ambientales ha sido de gran utilidad para diferentes programas de prevención. El uso del análisis geoespacial ha identificado zonas específicas en donde se concentra una mayor proporción de eventos de tránsito, como es el caso de zonas dedicadas a actividades comerciales y servicios 54 , o en las que diferentes usuarios de la vía pública, como los peatones 55 , tienen mayor riesgo de lesionarse 56 . Estas zonas son potencialmente modificables a través de intervenciones concretas 57,58 . Las auditorías viales tienen como propósito verificar que el diseño de las vialidades sea consistente con los principios de seguridad vial o la necesidad de cambios en su diseño para prevenir la siniestralidad ${ }^{4}$. La evidencia sugiere cómo, sin modificar las conductas de riesgo, la severidad de las lesiones puede disminuir significativamente al readecuar la estructura vial 52 incluso con estrategias de bajo costo 43 .

En México, la respuesta al problema de los atropellamientos ha sido la construcción masiva de puentes peatonales 35 . Es sabido que los puentes peatonales buscan favorecer la circulación de vehículos de motor y no ofrecer mayor seguridad a los peatones, de ahí que poco tomen en cuenta sus necesidades particulares. Así, los adultos mayores, las mujeres con niños y las personas con ciertos niveles de discapacidad, tienen dificultad para subir una escalera 34 o, en el mejor de los casos, las rampas construidas para tal efecto. Los peatones que sí podrían utilizar esos puentes no lo hacen, porque la misma estructura les permite cruzar las vialidades por abajo, lo que desincentiva esta conducta de seguridad vial. Se han documentado problemas relacionados con la inseguridad, lo que en conjunto motiva a los peatones a intercambiar el riesgo de utilizar un puente peatonal por el de cruzar a mitad de calle 35,59 .

\section{- Promoción de transporte sostenible}

Según la OMS, un sistema de tránsito seguro es aquél que "se adapta y contrarresta la vulnerabilidad y falibilidad humanas" 4 . Para ello recomienda fomentar el uso de medios de transporte más seguros, sostenibles y accesibles ${ }^{4}$. Esto incluye la promoción de medidas que estimulen el desplazamiento a pie o en bicicleta y el uso 
de transporte público masivo ${ }^{32}$. El éxito reciente que ha tenido la promoción de la bicicleta, como medio de transporte en ciertas ciudades del país, debe ser acompañado de cambios a la estructura que ofrezcan protección a este usuario. La construcción de ciclovías en la Ciudad de México, Guadalajara y León, así como la inversión en transporte público masivo en autobuses es un paso importante en materia de seguridad vial y promoción de transporte sostenible.

El sistema de transporte público masivo representa una opción de transporte rápida y eficiente que además tiene beneficios ambientales y sociales. Se han documentado mejoras en cuanto a tiempo y costo de viaje y emisiones de gases de efecto invernadero y contaminantes del aire 60 . De igual forma, se ha documentado una disminución en el número de LCT, a raíz de la puesta en marcha de sistemas de transporte público masivo en autobuses 61 . Entre las ciudades Mexicanas que cuentan con estos sistemas se encuentran la Ciudad de México (Metrobús), la ciudad de León (Optibus) y la ciudad de Guadalajara (Macrobús).

\section{- Legislación y seguridad vial}

La OMS ha llamado la atención sobre la importancia de legislar acerca de los principales factores de riesgo para controlar y limitar el problema de las LCT 62. México cuenta con legislación que prohíbe el consumo de alcohol al conducir, pero con excepción del estado de Jalisco, en la mayoría de las ciudades y entidades federativas del país, el nivel permitido de alcohol en sangre al conducir es de 0,08g/dL y es igual para adolescentes y adultos, en contra de las recomendaciones internacionales. Esto implica que no se basa en evidencia y suele ser más tolerante de lo ideal 32 . En este sentido, la aplicación de la legislación, ha sido calificada como reprobatoria 63 . De hecho, un estudio mostró que sólo un $36 \%$ de los conductores piensa que serán arrestados mientras manejan bajo la influencia del alcohol 23. Una de las limitaciones que impiden su correcta aplicación es que la propia legislación no habilita a las autoridades realizar detecciones aleatorias, componente fundamental para el éxito de estas estrategias 64 , aunque existen algunas excepciones exitosas (Figura 4).

México también cuenta con legislación que obliga el uso de cinturón de seguridad y SRI en todos los pasajeros, aunque su aplicación ha sido calificada como muy baja 32 . El uso de casco en motociclistas es obligatorio 32 ; sin embargo, la aplicación de la legislación también es baja y no existe regulación sobre la importancia de que el casco utilizado sea certificado 65 . Esto cuestiona la protección potencial que estos dispositivos pueden ofrecer. Dado que es muy probable que este actor vial incremente su peso relativo en el futuro, las estrategias nacionales deberían anticiparse a estos cambios.

Existe legislación que establece los límites de velocidad, tanto del ámbito federal, como local. Sin embargo, en el país, como en la región de las Américas, es un factor de riesgo al que poca atención se le presta 63 . Su aplicación es reprobatoria y pocas intervenciones se han implementado sistemáticamente para prevenir la exposición a velocidades inadecuadas. Una de ellas es la instalación de radares para la foto-infracción en Guadalajara que ha mostrado buenos resultados (Celis A. Comunicación personal; 2011). A pesar de ello, el uso de esta tecnología ha sido poco diseminado en el país. y durante los fines de semana, el Gobierno de la Ciudad de México puso en marcha el programa Conduce Sin Alcohol, iniciándolo el 19 de septiembre de 2003. Este programa consiste en la implementación de operativos permanentes de alcoholimetría y, en caso de mostrar un resultado positivo, se procede a un arresto administrativo inconmutable de 20 a 36 horas. Según las autoridades del Gobierno de la Ciudad de México, ha logrado reducir 30\% el número de muertes en eventos de tránsito relacionadas con la conducción bajo el influjo del alcohol. Tan sólo en el año previo, se implementaron 81 jornadas nocturnas y se evaluó a 244.883 conductores. En 31.699 de los casos se realizaron pruebas de alcoholimetría, un $27 \%$ de las cuales dieron resultados positivos 88 . El establecimiento de un marco jurídico previo a la implementación del programa que permitiera las detecciones aleatorias fue clave y facilitó la aplicación de la legislación, a cargo de la Secretaría de Seguridad Pública del Gobierno del Distrito Federal. 


\section{Discusión}

El elevado número de lesiones y muertes causadas por el tránsito posiciona al país, según algunos reportes, por encima del promedio en las Américas, sólo por debajo de Venezuela ${ }^{32}$. La coyuntura que ofrece el Decenio de Acción por la seguridad vial, representa una oportunidad inigualable de trabajar en aras de lograr controlar y abatir este importante problema de salud pública. La Estrategia Nacional de Seguridad Vial es un gran paso hacia la reducción de LCT. Sin embargo, esta estrategia debe ir acompañada de recursos financieros que permitan su realización. Para ello se requiere de un plan nacional, con estrategias claras y metas que puedan ser medibles en su evolución y establezca compromisos concretos para las distintas instituciones y para los tres niveles de gobierno.

Aunque México cuenta con legislación sobre los principales factores de riesgo, es evidente que no es integral. Una legislación, para ser integral, debe existir, ser adecuada y debe aplicarse estrictamente ${ }^{63}$. La legislación mexicana es aún perfectible pues se aplica poco 32 y aún no alcanza a algunos usuarios menos visibles, como los ciclistas. Un reto para el país es implementar una estrategia contra la conducción bajo el efecto del alcohol y otras drogas que implique la reducción de los niveles permitidos de alcohol, al menos a $0,04 \mathrm{~g} / \mathrm{dL}$ para adultos 4 y tolerancia cero en menores de 21 años y conductores inexpertos 66. Esta legislación deberá proveer un marco jurídico adecuado para que las autoridades correspondientes puedan aplicar detecciones aleatorias de alcohol en el país, estrategia que ha mostrado ser altamente costo-efectiva 32 . Esta estrategia deberá generar sinergias con políticas públicas que busquen reducir el consumo de alcohol, pues tienen externalidades positivas en seguridad vial 32,66 . Esta estrategia debe incluir a ciclistas y motociclistas pues también ellos están expuestos según muestra la ENSANut-2012. Por otro lado, se deben actualizar las normas que regulan la fabricación, importación y comercialización de cascos con estándares de protección adecuados, procurando que sean financieramente accesibles 67 . De igual forma, se debe unificar y mejorar el entrenamiento, la capacitación y el proceso para el otorgamiento de licencias para conducir. Esto podría incluir estrategias de licenciamiento gradual, especialmente en adolescentes 68 .

Para que la estrategia en seguridad vial sea efectiva, es necesario contar con información de calidad y oportuna. Un aspecto clave es establecer un sistema de vigilancia epidemiológica de las LCT, que incluya la atención pre-hospitalaria, hospitalaria y rehabilitación, y hallazgos de ser- vicio médico-forense, involucrando a todas las instituciones del país. Idealmente, esta información debería integrarse por individuo y debería poder ligarse a información obtenida por la policía de tránsito. Esto permitiría conocer, caso por caso, datos históricos y la evolución completa del evento de interés. Además, este sistema debería realizar un diagnóstico sobre discapacidad y secuelas de LCT (Figura 5).

Invertir en investigación es igualmente prioritario. Existen áreas que podrían verse beneficiadas con resultados de investigación específicos y evidencias para la toma de decisiones (Figura 6). La diversidad de resultados de investigación, que en ocasiones parecen contradictorios, resalta la importancia del contexto sobre este problema particular. La evidencia científica tiene poco sentido para la toma de decisiones, si no se adapta a las circunstancias del lugar donde se aplicará: la evidencia de lo que funciona debe ser combinada con la evidencia del contexto. El rol de la ciencia (sin considerar el contexto) es indicar lo que sabemos funciona en general; en el caso de la guía que considera al contexto es iluminar los dos: lo que funciona o no y cómo debe ser implementado en circunstancias específicas bajo consideración ${ }^{69}$. Incorporar la perspectiva de la comunidad permite identificar la problemática local y priorizar estrategias de prevención sensibles al contexto y culturalmente aceptadas 70 .

Abordajes que han mostrado ser efectivos en países de altos ingresos pueden no dar los mismos resultados favorables en países en desarrollo. Por ejemplo, una ley sobre el uso del cinturón de seguridad puede proteger a los conductores y pasajeros, pero dicha ley tendrá poco impacto en un país cuyo problema mayor sean los atropellamientos 71 . En virtud de que las intervenciones en salud pública tienden a ser complejas, programáticas, y a depender del contexto, si una intervención no funciona debemos determinar si la intervención falló (falla inherente a la intervención) o si fue una falla en su implementación 72 .

No todas las políticas de salud encaminadas a prevenir las LCT son benéficas para todos los usuarios, y algunas evidencias sugieren que los más vulnerables son los que se benefician menos. Dado que en México, la mayor parte de la evidencia generada se centra en ocupantes de vehículos automotores, no extraña que la mayoría de las intervenciones se haya centrado en este tipo de actor y poco en el respeto de los demás actores viales, lo que aumenta la desigualdad y vulnerabilidad de éstos 24 . Aunque el tema de la equidad está presente en la estrategia nacional, poco énfasis se hace en los mecanismos que permitirán disminuir las brechas existentes. Por 
Figura 5

Atención médica pre-hospitalaria y de urgencias.

El quinto pilar de la Década de Acción por la Seguridad Vial tiene que ver con la respuesta del sistema de salud, cuando las lesiones causadas por el tránsito se han presentado. Este pilar busca incrementar la respuesta a las emergencias, con el objetivo de mejorar la habilidad del sistema de salud para proveer atención de pre-hospitalaria y hospitalaria apropiada y oportuna, así como la subsecuente rehabilitación en el largo plazo. A pesar de que se ha resaltado su importancia, por ahora existe poca información secundaria en el país, en relación a la calidad de la atención médica de los lesionados desde el enfoque de la salud pública. Poca información es recabada de forma sistemática por los sistemas de información disponibles. Cuando existe esta información, el acceso suele estar restringido lo que complica el análisis subsecuente. Esto es particularmente evidente en el tema de la atención pre-hospitalaria.

En este sentido, Pinet 89 identifica serios problemas de coordinación, regulación y evaluación del desempeño de la atención prehospitalaria en la Ciudad de México. Algunos reportes recientes muestran que ha habido avances al respecto, que pudieran explicar el decremento observado en la mortalidad por atropellamientos en esta misma ciudad, como la creación del Sistema Integral de Urgencias Médicas ${ }^{90}$. Por otro lado, Mock et al. ${ }^{91}$ han documentado la experiencia de Monterrey a este respecto. Según este autor al menos $50 \%$ del personal que otorga atención pre-hospitalaria no cuenta con la capacitación adecuada. Las distintas estrategias de entrenamiento y capacitación que han mostrado resultados exitosos en otros contextos, no se han diseminado exitosamente en esta ciudad. La falta o deficiente cobertura de recursos tiene un impacto negativo, pues se ha observado que los tiempos promedio totales están por encima de lo ideal.

Figura 6

Algunas áreas de investigación prioritarias para México.

- Estimar la carga de las LCT que es atribuible directamente a los distintos factores de riesgo: alcohol y otras drogas, no uso de casco, uso de casco no certificado, uso de celular, no uso de sistemas de retención, entre otros;

- Evaluar y dar seguimiento al uso de dispositivos de seguridad (cinturón de seguridad, SRI, casco) y a la exposición a factores de riesgo:

velocidad, telefonía móvil, alcohol y conducción, etc. y la carga de las LCT que puede ser atribuida a cada uno de ellos;

- Evaluar constantemente el uso de otras drogas con el fin de observar su comportamiento en el tiempo;

- Realizar estudios sobre la calidad de la atención pre-hospitalaria, hospitalaria y de servicios de rehabilitación. Idealmente, estos estudios deberían incluir un análisis sobre las potenciales barreras de acceso a los diferentes servicios;

- Documentar el doble riesgo al que se enfrentan las personas que viven con discapacidad, tema pendiente en la agenda de investigación nacional e internacional 36 ;

- Realizar análisis diagnósticos sistemáticos de la estructura vial (auditorías viales), como estrategia para identificar necesidades de inversión en materia de infraestructura vial;

- Generar evidencias constantemente sobre el nivel de aplicación de la legislación vigente;

- Evaluar el impacto de diferentes intervenciones, programas e iniciativas nacionales y locales sobre el estado de salud, tema poco documentado en el contexto nacional. Incluir el análisis de la inversión en prevención de LCT y su efecto sobre la carga de estos eventos en el sistema de salud.

LCT: lesiones causadas por el tránsito; SRI: sistemas de retención infantil.

ejemplo, no es explícita la política de respeto al peatón y sus espacios.

Dado el alto número de peatones, ciclistas y motociclistas que se lesionan y mueren, la estrategia debería estar equilibrada de tal manera que atienda las necesidades de todos los usuarios. Como se ha enfatizado con anterioridad, de acuerdo al tipo de usuario, se pueden identificar problemáticas diferentes $\mathrm{y}$, por tanto, distintas alternativas de solución. De ahí que se requiere un cambio de paradigma para dar igual protección a todos los usuarios, para que las calles y las autopistas no produzcan una carga injusta de lesiones y muerte en los grupos más vulnerables 73 
En este sentido, se debe valorar la inclusión y evaluación, como parte de la estrategia de seguridad vial, de medidas que incrementen la visibilidad de los usuarios de la vía pública: obligatoriedad del uso de las luces diurnas para vehículos de motor; uso de ropa, accesorios y vehículos de colores brillantes que mejoren la visibilidad de los peatones, ciclistas y motociclistas 74 . De igual forma, se debe promover el uso obligatorio de casco en ciclistas.

Podemos concluir que existen aún retos y oportunidades en el tema de prevención de las LCT. Al ser un problema complejo y multicausal que atañe a diferentes sectores y disciplinas, un aspecto importante de resaltar es que su manejo y prevención requieren de abordajes multisectoriales. Así, la prevención de las consecuencias de las LCT es el resultado de la definición de responsabilidades que atañen a cada sector en particular y del trabajo intersectorial coordinado con objetivos claramente definidos y comunes a todos ${ }^{3}$. Un primer paso es reconocer en las
LCT un problema urgente de salud pública que afecta de forma diferenciada a cada uno de los usuarios de la vía pública. En términos de políticas públicas se requiere incrementar el uso de sistemas de retención y cascos, y disminuir la exposición al alcohol y drogas al conducir. Para ello se debe dedicar especial atención a la actualización de la legislación y su estricta aplicación. También se debería fortalecer las capacidades de los gobiernos para garantizar e impulsar la movilidad de forma segura, equitativa, saludable y sostenible para todos los actores viales. Una movilidad segura implica considerar las necesidades de todos los actores de la vía pública, y en especial, de los más vulnerables. Para dar respuesta a los acuerdos establecidos en la Estrategia Nacional de Seguridad Vial 2011-2020 y reducir las lesiones, discapacidades y muertes causadas por el tránsito, se deberá continuar, ampliar y fortalecer la Iniciativa Mexicana de Seguridad Vial (IMESEVI).

\section{Resumo}

Por meio da revisão oficial de informações secundárias, publicações científicas e da análise da ENSANut 2012, este trabalho visa a proporcionar uma visão abrangente da magnitude atual das lesões causadas pelo trânsito no México e analisar criticamente a resposta social organizada. Os altos índices de acidentes, lesões, mortes e invalidez atribuídos a esse problema de saúde pública representam um alto custo para a sociedade mexicana, especialmente para as famílias. Discutimos neste estudo como a resposta mexicana tem focado em motoristas, deixando de fora os usuários vulneráveis das vias, e tem favorecido a implementação de estratégias pouco efetivas. Embora exista legislação que abrange os principais fatores de risco, tem sido negligenciada a sua correta aplicação. A estratégia mexicana poderia ser fortalecida por meio da implementação de estratégias de mobilidade segura, equitativa, saudável e sustentável para os diferentes atores da vias. Apesar dos grandes avanços que foram feitos em termos de prevenção das lesões causadas pelo trânsito, ainda existem desafios e oportunidades para o país.

\section{Colaboradores}

R. Pérez-Núñez participó de la concepción del estudio, análisis, redacción y aprobación de la versión final. M. Híjar, A. Celis y E. Hidalgo-Solórzano participaron de la concepción del estudio, análisis, revisión crítica del documento y aprobación de la versión final.

\section{Agradecimientos}

Se agradece el apoyo brindado por Juan Daniel Vera López y Lourdes Gómez García para la preparación de este documento; así como los comentarios recibidos por el grupo de análisis de la ENSANut-2012 y, particularmente, al Dr. Adolfo Martínez Valle por comentar y retroalimentar versiones previas de este reporte. Se agradece el apoyo brindado por la Dirección General Adjunta de Epidemiología y la Dirección de Información en Salud por facilitarnos información recopilada por sus dependencias. Agradecemos los comentarios recibidos por los pares revisores de la revista Cadernos de Saúde Pública. 


\section{Referencias}

1. Lozano R, Naghavi M, Foreman K, Lim S, Shibuya $\mathrm{K}$, Aboyans V, et al. Global and regional mortality from 235 causes of death for 20 age groups in 1990 and 2010: a systematic analysis for the Global Burden of Disease Study 2010. Lancet 2012; 380: 2095-128.

2. World Health Organization. Global status report on road safety 2013: supporting a decade of action. Geneva: World Health Organization; 2013.

3. Híjar-Medina M, Vázquez-Vela E. Foro nacional sobre accidentes de tránsito en México. Enfrentando los retos a través de una visión intersectorial. Cuernavaca: Instituto Nacional de Salud Pública; 2003.

4. Peden MM, Scurfield R, Mohan D, Hyder AA, Jarawan $\mathrm{E}$, Mathers $\mathrm{C}$. World report on road traffic injury prevention. Geneva: World Health Organization; 2004

5. Norton R, Kobusingye O. Injuries. N Engl J Med 2013; 368:1723-30.

6. Romero-Martínez M, Shamah-Levy T, Franco A, Villalpando S, Cuevas-Nasu L, Gutiérrez J, et al. Encuesta Nacional de Salud y Nutrición 2012: diseño y cobertura. Salud Pública Mex 2013; 55 Suppl 2:S332-40

7. Gutiérrez J, Rivera-Dommarco J, Shamah-Levy T, Villalpando-Hernández S, Franco A, Cuevas-Nasu L, et al. Encuesta Nacional de Salud y Nutrición 2012. Resultados nacionales. Cuernavaca: Instituto Nacional de Salud Pública; 2012.

8. Pérez-Núñez R, Híjar M, Celis A, Hidalgo-Solórzano E. Lesiones causadas por el tránsito: hora de poner el freno. Cuernavaca: Instituto Nacional de Salud Pública; 2012.

9. Hijar M, Chandran A, Pérez-Núñez R, Lunnen JC Rodriguez-Hernandez JM, Hyder AA. Quantifying the underestimated burden of road traffic mortality in Mexico: a comparison of three approaches. Traffic Inj Prev 2012; 13 Suppl 1:5-10.

10. Shahraz S, Bhalla K, Lozano R, Bartels D, Murray CJ. Improving the quality of road injury statistics by using regression models to redistribute ill-defined events. Inj Prev 2013; 19:1-5.

11. Bartels D, Bhalla K, Shahraz S, Abraham J, Lozano $\mathrm{R}$, Murray CJ. Incidence of road injuries in Mexico: country report. Int J Inj Contr Saf Promot 2010 17:169-76.

12. Juárez-Adauta S, Ávila-Burgos L. Atención en servicios de urgencias a pacientes lesionados en vía pública. Rev Med Inst Mex Seguro Soc 2006; 44:433-40

13. Hidalgo-Solorzano EC, Hjar M, Blanco-Munoz J, Kageyama-Escobar ML. Factors related with the severity of injuries in public streets of an urban area in Cuernavaca, Morelos, Mexico. Salud Pública Méx 2005; 47:30-8.

14. Ávila-Burgos L, Ventura-Alfaro CE, Hidalgo-Solorzano EC, Hijar-Medina M, Aracena-Genao B, Celis-de la Rosa AJ. Atención de lesiones por tipo de causa externa en salas de urgencia en tres ciudades de México: Composición, frecuencia y gravedad. Rev Invest Clín 2012; 64:336-43.
15. Ávila-Burgos L, Medina-Solis CE, Pérez-Núñez R, Hijar-Medina M, Aracena-Genao B, Hidalgo-Solorzano E, et al. Prevalencia de accidentes de tránsito no fatales en México: resultados de la ENSANUT 2006. Salud Pública Méx 2008; 50 Suppl 1:S38-47.

16. Bhalla K, Shahraz S, Naghavi M, Lozano R, Murray C. Estimating the distribution of external causes in hospital data from injury diagnosis. Accid Anal Prev 2008; 40:1822-9.

17. Ávila-Burgos L, Ventura-Alfaro C, Barroso-Quiab A, Aracena-Genao B, Cahuana-Hurtado L, ServánMori E, et al. Las lesiones por causa externa en México. Lecciones aprendidas y desafíos para el Sistema Nacional de Salud. Cuernavaca: Instituto Nacional de Salud Pública; 2010.

18. Valdespino JL, Olaiz G, López-Barajas MP, Mendoza L, Palma O, Velázquez O, et al. Encuesta Nacional de Salud 2000. Tomo I. Vivienda, población y utilización de servicios de salud. Cuernavaca: Instituto Nacional de Salud Pública; 2003.

19. Centro Nacional para la Prevención de Accidentes. Iniciativa Mexicana de Seguridad Vial y Prevención de Lesiones en el Tránsito (IMESEVI). Construcción de línea base. Reporte final. México DF: Secretaría de Salud; 2009.

20. Cuevas-Colunga AC, Villegas-Villegas N, MayoralGrajeda EF, Mendoza-Díaz A. Anuario estadístico de accidentes en carreteras federales 2009. Sanfandila: Instituto Mexicano del Transporte, Secretaría de Comunicaciones y Transportes; 2010.

21. Arreola-Rissa C, Santos-Guzman J, Esquivel-Guzman A, Mock CN. Traffic related deaths in Nuevo Leon, Mexico: causes and associated factors. Salud Pública Méx 2008; 50 Suppl 1:S48-54.

22. Hijar M, Carrillo C, Flores M, Anaya R, Lopez V. Risk factors in highway traffic accidents: a case control study. Accid Anal Prev 2000; 32:703-9.

23. Hijar M, Flores M, Lopez MV, Rosovsky H. Alcohol intake and severity of injuries on highways in Mexico: a comparative analysis. Addiction 1998; 93:1543-51.

24. Híjar M. El crecimiento urbano y sus consecuencias no planeadas. El caso de los atropellamientos. In: Knaul F, Nigenda G, editors. Caleidoscopio de la salud. De la investigación a las políticas y de las políticas a la acción. México DF: Funsalud; 2003. p. 89-97.

25. Olaiz-Fernández G, Rivera-Dommarco J, ShamahLevy T, Rojas R, Villalpando-Hernández S, Hernández-Ávila M, et al. Encuesta Nacional de Salud y Nutrición 2006. Cuernavaca: Instituto Nacional de Salud Pública; 2006.

26. Pérez-Núñez R, Chandran A, Hijar M, Celis A, Carmona-Lozano MS, Lunnen JC, et al. The use of seatbelts and child restraints in three Mexican cities. Int J Inj Contr Saf Promot 2013; 20:385-93.

27. Mock C, Arreola Rissa C, Trevino Perez R, Almazan Saavedra V, Enrique Zozaya J, Gonzalez Solis R, et al. Childhood injury prevention practices by parents in Mexico. Inj Prev 2002; 8:303-5.

28. Chandran A, Lunnen JC, Pérez-Núñez R, Hijar M, Hidalgo-Solorzano Edel C, Hyder AA. The prevalence of motorcicle helmet use in three Mexican cities. Inj Prev 2012; 18 Suppl 1:A233. 
29. Hidalgo-Solorzano EC, Inclán-Valadez C, PérezNúñez R, Hijar M. Motorcicle non-standard helmet use in an urban area of Mexico. Inj Prev 2012; 18 Suppl 1:A192.

30. Vera-Lopez JD, Pérez-Núñez R, Hijar M, HidalgoSolorzano E, Lunnen JC, Chandran A, et al. Distracted driving: mobile phone use while driving in three Mexican cities. Inj Prev 2013; 19:276-9.

31. Pérez-Núñez R, Hidalgo-Solorzano E, Vera-Lopez JD, Lunnen JC, Chandran A, Hijar M, et al. The prevalence of mobile phone use among motorcyclists in three Mexican cities. Traffic Inj Prev 2014 15:148-50.

32. Organización Panamericana de la Salud. Informe sobre el estado de la seguridad vial en la región de las Américas. Washington DC: Organización Panamericana de la Salud; 2009.

33. Celis A, Gomez Z, Martinez-Sotomayor A, Arcila L, Villasenor M. Family characteristics and pedestrian injury risk in Mexican children. Inj Prev 2003; 9:58-61.

34. Hijar M, Trostle J, Bronfman M. Pedestrian injuries in Mexico: a multi-method approach. Soc Sci Med 2003; 57:2149-59.

35. Hidalgo-Solorzano E, Campuzano-Rincón J, Rodríguez-Hernández JM, Chias-Becerril L, Resendiz-Lopez H, Sanchez-Restrepo H, et al. Motivos de uso y no uso de puentes peatonales en la Ciudad de México: la perspectiva de los peatones. Salud Pública Méx 2010; 52:502-10.

36. Shakespeare T. Double jeopardy? Disability and road traffic injury. Road Traffic Injuries Research Network Newsletter 2011; (2):2-3.

37. Inclan C, Hijar M, Tovar V. Social capital in settings with a high concentration of road traffic injuries. The case of Cuernavaca, Mexico. Soc Sci Med 2005; 61:2007-17.

38. Ávila-Burgos L, Aracena-Genao B, Ventura-Alfaro C, Barroso-Quiab CA. Consecuencias económicas de las lesiones por accidente de tránsito para el sistema de salud mexicano. In: 14 Congreso de Investigación en Salud Pública. Cuernavaca: Instituto Nacional de Salud Pública; 2011. p. 75.

39. Pérez-Núñez R, Avila-Burgos L, Hijar-Medina M, Pelcastre-Villafuerte B, Celis A, Salinas-Rodriguez A. Economic impact of fatal and non-fatal road traffic injuries in Guadalajara Metropolitan Area and Jalisco, Mexico. Inj Prev 2011; 17:297-303.

40. World Health Organization. Investing in heatlh: a full report of the findings of the Commission on Macroeconomics and Health. Geneva: World Health Organization; 2003.

41. Pérez-Núñez R, Pelcastre-Villafuerte B, Hijar M, Avila-Burgos L, Celis A. A qualitative approach to the intangible cost of road traffic injuries. Int J Inj Contr Saf Promot 2012; 19:69-79.

42. Hijar M, Vazquez-Vela E, Arreola-Risa C. Pedestrian traffic injuries in Mexico: a country update. Inj Control Saf Promot 2003; 10:37-43.

43. Arreola-Rissa C, Santos-Guzmán J, Esquivel-Guzmán A, Mock C, Herrera-Escamilla A. Barriles de absorción y contención del impacto: reducción de mortalidad por accidentes de tránsito. Salud Pública Méx 2008; 50 Suppl 1:S55-9.
44. Haddon Jr. W. The changing approach to the epidemiology, prevention, and amelioration of trauma: the transition to approaches etiologically rather than descriptively based. Am J Public Health Nations Health 1968; 58:1431-8.

45. Robertson LS, Kelley AB, O'Neill B, Wixom CW, Eiswirth RS, Haddon Jr. W. A controlled study of the effect of television messages on safety belt use. Am J Public Health 1974; 64:1071-80.

46. Doyle YG, Furey A, Flowers J. Sick individuals and sick populations: 20 years later. J Epidemiol Community Health 2006; 60:396-8.

47. Rose G. Sick individuals and sick populations. Int J Epidemiol 1985; 14:32-8.

48. Ajzen I. The theory of planned behavior. Organ Behav Hum Decis Process 1991; 50:179-211.

49. Evans D, Norman P. Predicting adolescent pedestrians' road-crossing intentions: an application and extension of the Theory of Planned Behaviour. Health Educ Res 2003; 18:267-77.

50. Lorenc T, Petticrew M, Welch V, Tugwell P. What types of interventions generate inequalities? Evidence from systematic reviews. J Epidemiol Community Health 2012; 67:190-3.

51. Recommendations to reduce injuries to motor vehicle occupants: increasing child safety seat use increasing safety belt use, and reducing alcoholimpaired driving. Am J Prev Med 2001; 21(4 Suppl):16-22.

52. Híjar-Medina MC, Carrillo-Ordaz CE, Flores-Aldana ME, Anaya R, López-López MV. Factores de riesgo de lesión por accidentes de tráfico y el impacto de una intervención sobre la carretera. Rev Saúde Pública 1999; 33:505-12.

53. WHO Regional Office for South-East Asia. Regional report on status of road safety. The South-East Asia region: a call for policy direction. New Delhi: WHO Regional Office for South-East Asia; 2009.

54. Hernández Hernández V. Análisis exploratorio espacial de los accidentes de tránsito en Ciudad Juárez, México. Rev Panam Salud Pública 2012; 31:396-402.

55. Hijar MC, Kraus JF, Tovar V, Carrillo C. Analysis of fatal pedestrian injuries in Mexico City, 1994-1997. Injury 2001; 32:279-84.

56. Híjar-Medina MC. Utilidad del análisis geográfico en el estudio de las muertes por atropellamiento. Salud Pública Méx 2000; 42:188-93.

57. Roberts I, Norton R, Jackson R, Dunn R, Hassall I. Effect of environmental factors on risk of injury of child pedestrians by motor vehicles: a case-control study. BMJ 1995; 310:91-4.

58. Agran PF, Winn DG, Anderson CL, Tran C, Del Valle CP. The role of the physical and traffic environment in child pedestrian injuries. Pediatrics 1996; 98(6 Pt 1):1096-103.

59. Ameratunga S, Hijar M, Norton R. Road-traffic injuries: confronting disparities to address a globalhealth problem. Lancet 2006; 367:1533-40.

60. Medina-Ramírez S. La importancia de reducción del uso del automóvil en México. Tendencias de motorización, del uso del automóvil y de sus impactos. México DF: Instituto de Políticas para el Transporte y el Desarrollo; 2012. 
61. Hidalgo D, Carrigan A. Modernización del transporte público. Washington DC: EMBARQ, World Resources Institute; 2010.

62. Mathers C, Fat DM, Boerma JT; World Health Organization. The global burden of disease: 2004 update. Geneva: World Health Organization; 2008.

63. Híjar M, Pérez-Núñez R, Inclán-Valadez C, Silveira-Rodrigues EM. Road safety legislation on the Americas. Rev Panam Salud Pública 2012; 32:70-6.

64. Global status report on road safety: time for action. Geneva: World Health Organization; 2009.

65. Hidalgo-Solórzano E. Uso de casco en motociclistas: un estudio en la zona urbana de Cuernavaca, México. In: 14o Congreso de Investigación en Salud Pública. Cuernavaca: Instituto Nacional de Salud Pública; 2011.

66. Organización Mundial de la Salud. Estrategia mundial para reducir el uso nocivo del alcohol Geneva: Organización Mundial de la Salud; 2010.

67. Ackaah W, Afukaar F, Agyemang W, Thuy Anh T, Hejar AR, Abdul G, et al. The use of non-standard motorcycle helmets in low- and middle-income countries: a multicentre study. Inj Prev 2013; 19:158-63.

68. Zhu M, Cummings P, Chu H, Coben JH, Li G. Graduated driver licensing and motor vehicle crashes involving teenage drivers: an exploratory agestratified meta-analysis. Inj Prev 2013; 19:49-57.

69. Lomas J, Culyer T, McCutcheon C, McAuley L, Law S. Conceptualizing and combining evidence for health system guidance (final report). Ottawa: Canadian Health Services Research Foundation 2005.

70. Trevino-Siller S, Hijar M, Mora G. Prioritisation of road traffic injury interventions: results of a participative research with stakeholders in Mexico. Int J Inj Contr Saf Promot 2011; 18:219-25.

71. Nantulya VM, Sleet DA, Reich MR, Rosenberg M, Peden M, Waxweiler R. Introduction: the global challenge of road traffic injuries: can we achieve equity in safety? Inj Control Saf Promot 2003; 10: 3-7.

72. Rychetnik L, Frommer M, Hawe P, Shiell A. Criteria for evaluating evidence on public health interventions. J Epidemiol Community Health 2002; 56:119-27.

73. Nantulya VM, Reich MR. Equity dimensions of road traffic injuries in low- and middle-income countries. Inj Control Saf Promot 2003; 10:13-20.

74. Mohan D, Tiwari G, Khaayesi M, Nafunkho FM. Prevención de lesiones causadas por el tránsito. Manual de capacitación. Washington DC: Organización Panamericana de la Salud; 2008.

75. Elvik R, Vaa T. The handbook of road safety measures. Amsterdam: Elsevier Science; 2004.

76. Zhu M, Cummings P, Chu H, Cook LJ. Association of rear seat safety belt use with death in a traffic crash: a matched cohort study. Inj Prev 2007; 13:183-5.

77. Zaza S, Sleet DA, Thompson RS, Sosin DM, Bolen JC. Reviews of evidence regarding interventions to increase use of child safety seats. Am J Prev Med 2001; 21(4 Suppl):31-47.
78. Liu BC, Ivers R, Norton R, Boufous S, Blows S, Lo SK. Helmets for preventing injury in motorcycle riders. Cochrane Database Syst Rev 2008; (1):CD004333.

79. Crompton JG, Bone C, Oyetunji T, Pollack KM, Bolorunduro O, Villegas C, et al. Motorcycle helmets associated with lower risk of cervical spine injury: debunking the myth. J Am Coll Surg 2011; 212: 295-300.

80. Peek-Asa C, McArthur DL, Kraus JF. The prevalence of non-standard helmet use and head injuries among motorcycle riders. Accid Anal Prev 1999; 31:229-33.

81. Redelmeier DA, Tibshirani RJ. Association between cellular-telephone calls and motor vehicle collisions. N Engl J Med 1997; 336:453-8.

82. McEvoy SP, Stevenson MR, McCartt AT, Woodward M, Haworth C, Palamara P, et al. Role of mobile phones in motor vehicle crashes resulting in hospital attendance: a case-crossover study. BM] 2005; 331:428

83. Wilson FA, Stimpson JP. Trends in fatalities from distracted driving in the United States, 1999 to 2008. Am J Public Health 2010; 100:2213-9.

84. Hatfield J, Murphy S. The effects of mobile phone use on pedestrian crossing behaviour at signalized and unsignalized intersections. Accid Anal Prev 2007; 39:197-205.

85. Mohan D. Road safety in less-motorized environments: future concerns. Int J Epidemiol 2002; 31:527-32.

86. LaScala EA, Gruenewald PJ, Johnson FW. An ecological study of the locations of schools and child pedestrian injury collisions. Accid Anal Prev 2004; 36:569-76.

87. Secretaría de Comunicaciones y Transportes. Acuerdo por el que se da a conocer la Estrategia Nacional de Seguridad Vial 2011-2020. Diario Oficial de la Federación. Órgano del Gobierno Constitucional de los Estados Unidos Mexicanos 2011; número 4, sección 1. Tomo DCXCIII. http://dof.gob. $\mathrm{mx} /$ nota_detalle.php? codigo $=5193284 \&$ fecha $=$ 06/06/2011.

88. Gobierno del Distrito Federal. 6to. Informe de Gobierno del Distrito Federal 2011-2012. México DF: Corporación Mexicana de Impresión; 2012. p. 152-3.

89. Pinet LM. Atención pre-hospitalaria de urgencias en el Distrito Federal: las oportunidades del sistema de salud. Salud Pública Méx 2005; 47:64-71.

90. Rodríguez-Hernández JM, Campuzano-Rincón JC, Hijar M. Comparación de datos sobre mortalidad por atropellamientos en la Ciudad de México: ¿se han presentado cambios en una década? Salud Pública Méx 2011; 53:320-8.

91. Mock C, Arreola-Risa C, Quansah R. Strengthening care for injured persons in less developed countries: a case study of Ghana and Mexico. Inj Control Saf Promot 2003; 10:45-51.

Recibido el 08/Feb/2013

Versión final presentada el 12/Ago/2013

Aprobado el 10/Feb/2014 